\title{
Accurate calibration and optimized measurement: use of an achromatic compensator in rotating polarizer spectroscopic ellipsometry
}

University of Metz

L.P.L.I. - CP 87811 F-57078 METZ CEDEX 3 FRANCE

\begin{tabular}{|l}
\hline We describe an improvement of a Rotating Polarizer Ellipsometer in order to increase the sensitivity for \\
the measurement of a transparent sample. We added a compensator, namely a quasi-achromatic \\
retardation quarter wave plate on the $400-700 \mathrm{~nm}$ spectral range. This new configuration is named \\
Polarizer Rotating-Polarizer Compensator Spectroscopic Ellipsometer (PRPCSE). We present a method \\
for calibration and the advantages for measurement of the transparent materials. This method is applied \\
to $\mathrm{CuCl}$ nanocrystals embedded in a NaCl transparent matrix.
\end{tabular}

\section{Diagram of PRPCSE}

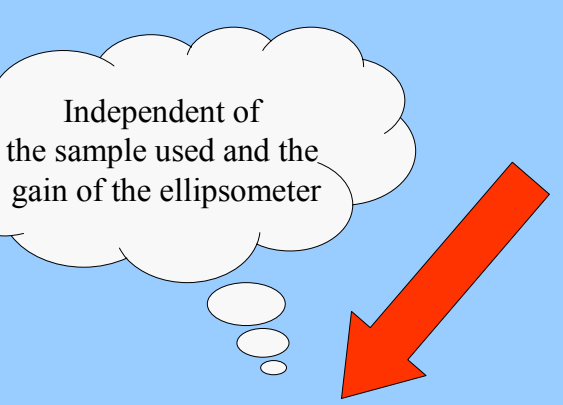

CALIBRATION

The methods for the calibration of the polarizers and analyzer are exactly the same as for the PRPSE ellipsometer. The PRPSE is the same configuration without the compensator.

The residue calibration for the compensator is a powerfull method because it is independent of the phase shift of the compensator.
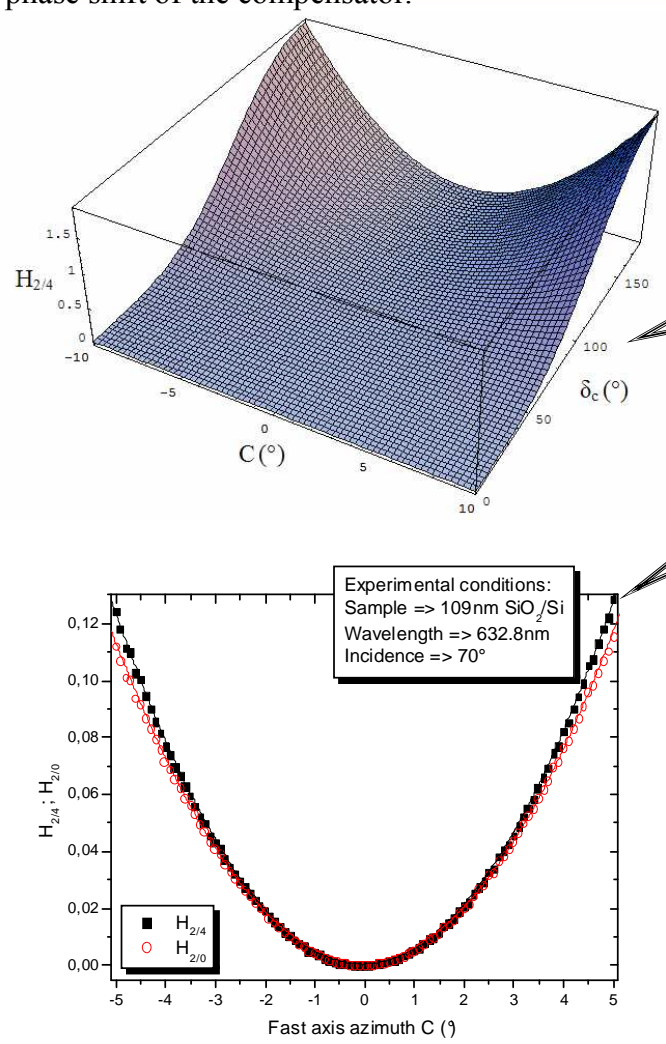

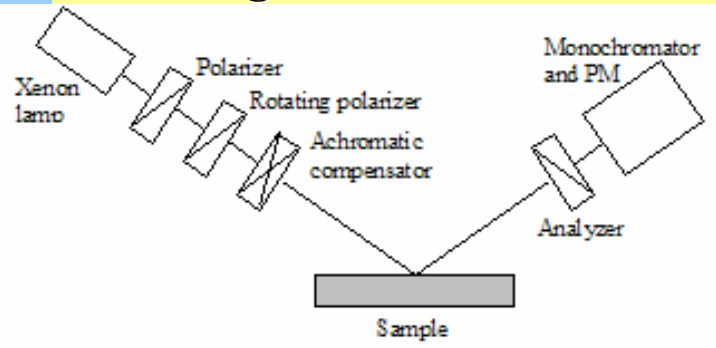

The detected photocurrent has the form :

$\mathrm{I}=\alpha_{0}+\alpha_{2 \mathrm{c}} \cos 2 \omega \mathrm{t}+\alpha_{2 \mathrm{~s}} \sin 2 \omega \mathrm{t}+\alpha_{4 \mathrm{c}} \cos 4 \omega \mathrm{t}+\alpha_{4 \mathrm{~s}} \sin 4 \omega \mathrm{t}$ where $\mathrm{P}_{\mathrm{t}}=\omega \mathrm{t}$ is the azimuth of the rotating polarizer.

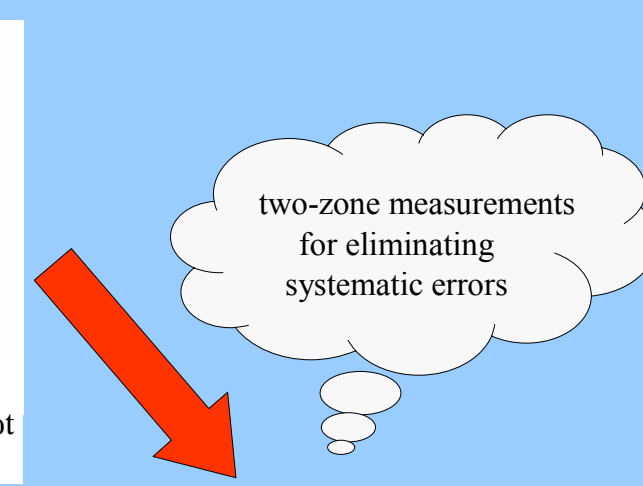

MEASUREMENT
This configuration allows to extract the ellipsometric parameters by using 3 sets of the analyzer and compensator positions.

The influence of the gain is eliminating by using the modified coefficients as $\beta_{\mathrm{i}}=\alpha_{\mathrm{i}} /\left(\alpha_{2 \mathrm{c}}-2 \alpha_{4 \mathrm{c}}\right)$

where $\mathrm{i}=2 \mathrm{c}, 2 \mathrm{~s}, 4 \mathrm{c}, 4 \mathrm{~s}$ and $\mathrm{P}=0^{\circ}$.

The average incident light irradiance is not used for the measurements.

Simulation of the ratio $\mathrm{H}_{2 / 4}=\left(\alpha_{2 \mathrm{c}}{ }^{2}+\alpha_{2 \mathrm{~s}}{ }^{2}\right) /\left(\alpha_{4 \mathrm{c}}^{2}+\alpha_{4 \mathrm{~s}}{ }^{2}\right)$ when the fast axis azimuth (C) is close to the plane of incidence. $\mathrm{H}_{2 / 4} \# 64 \mathrm{C}^{2} \sin ^{2}\left(\delta_{\mathrm{c}} / 2\right)$.

Experimental data for the calibration of the compensator $\left(\right.$ analyzer $=0^{\circ}$ and polarizer $\left.=90^{\circ}\right)$. 2 methods are possible: $\mathrm{H}_{2 / 4}$ or $\mathrm{H}_{2 / 0}$.

Experimental measurements of $\mathrm{CuCl}$ nanocrystals embedded in a $\mathrm{NaCl}$ transparent matrix

- The PRPSE configuration

(with optimized tracking method) allows to measure $\tan \Psi$ and $\cos \Delta$ (experimental errors are proportionnal to $\sin ^{-1} \Delta$ ).

- The PRPCSE configuration allows to measure $\tan \Psi$ and $\tan \Delta$ (experimental errors are proportionnal to $\cos ^{2} \Delta$ ).

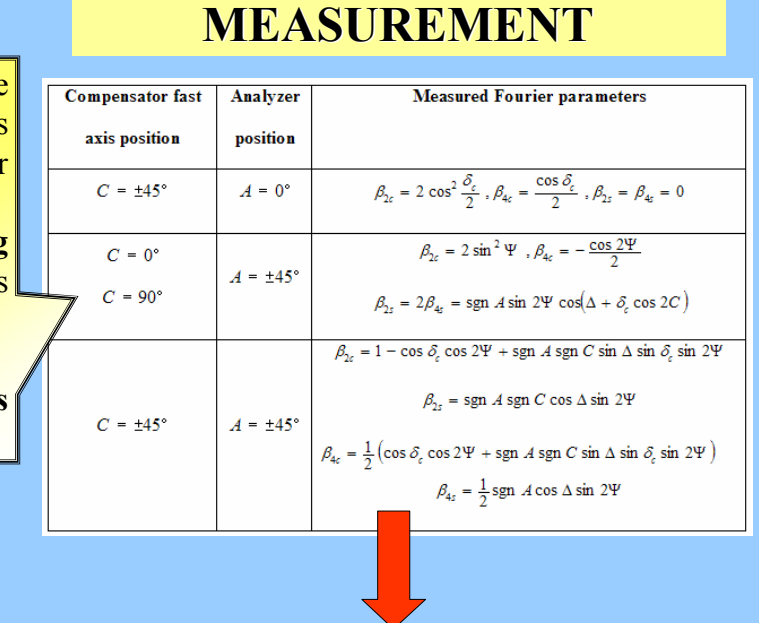

- From the first set of analyzer and compensator positions we deduce $\cos \delta_{\mathrm{c}}$.

- The second set allows to determine the parameter $\tan \Psi$ and if necessary $\cos \left(\Delta \pm \delta_{\mathrm{c}}\right)$.

- Finally the third set consists in determining $\tan \Delta$.

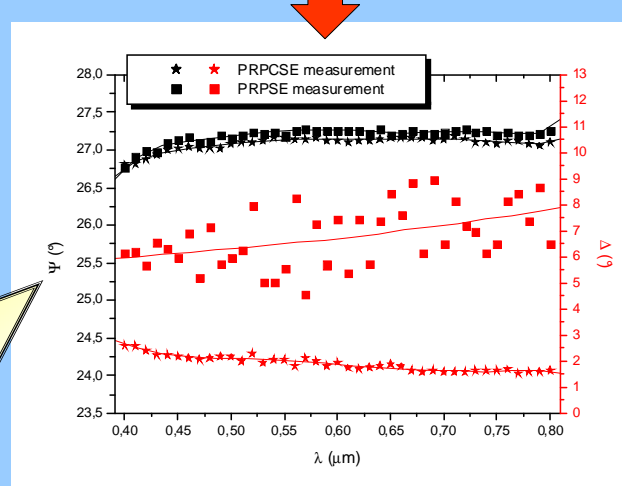

References:

[1] B. Lecourt, Ph. D. thesis, University of Bordeaux I, 1998.

[2] P. Chindaudom, K. Vedam, Applied Optics, 32 (1993) 6391.

[3] S. Kawabata, J. Opt. Soc. Am A 4, 664 (1987).

[4] A. En Naciri, L. Broch, L. Johann, R. Kleim, Thin Solid Films 406 (2002) 103.

[5] S. Bertucci, A. Pawlowski, N. Nicolas, L. Johann, A. El Ghemmaz, N. Stein, R. Kleim, Thin Solid Films 313-314 (1998) 73 